\title{
Dietary supplementation with methyl donors reduces fatty liver and modifies the fatty acid synthase DNA methylation profile in rats fed an obesogenic diet
}

\author{
P. Cordero • A. M. Gomez-Uriz • J. Campion • \\ F. I. Milagro $\cdot$ J. A. Martinez
}

Received: 28 February 2012/Accepted: 11 May 2012/Published online: 31 May 2012

(C) Springer-Verlag 2012

\begin{abstract}
Non-alcoholic fatty liver disease (NAFLD) is one of the first hepatic manifestations of metabolic syndrome, whose progression can lead to cirrhosis and hepatic carcinoma. Interestingly, methyl donor supplementation could improve obesogenic diet-induced hepatic triglyceride accumulation. The aim of this research is to describe methyl donor effects on a high-fat-sucrose (HFS) diet in both sexes and epigenetic changes induced on fatty acid synthase (FASN) promoter methylation pattern as well as gene expression of NAFLD key metabolic genes. Twenty-four male and 28 female Wistar rats were assigned to three dietary groups: control, HFS, and HFS supplemented with methyl donors (choline, betaine, vitamin B12, and folic acid). After 8 weeks of treatment, somatic, biochemical, mRNA, and epigenetic measurements were performed. Rats fed the HFS diet presented an overweight phenotype and alterations in plasma biochemical measurements. Methyl donor supplementation reverted the HFS-diet-induced hepatic triglyceride accumulation. Analysis of FASN promoter cytosine methylation showed changes in both sexes due to the obesogenic diet at $-1,096,-780,-778$, and $-774 \mathrm{CpG}$ sites with respect to the transcriptional start site. Methyl donor supplementation modified DNA methylation at $-852,-833,-829,-743$, and -733 CpGs depending on the sex. RT-PCR analysis confirmed that FASN expression
\end{abstract}

Electronic supplementary material The online version of this article (doi:10.1007/s12263-012-0300-z) contains supplementary material, which is available to authorized users.

P. Cordero - A. M. Gomez-Uriz · J. Campion ·

F. I. Milagro · J. A. Martinez $(\square)$

Department of Nutrition and Food Science,

Physiology and Toxicology, University of Navarra,

31008 Pamplona, Spain

e-mail: jalfmtz@unav.es tended to be altered in males. Our findings reinforce the hypothesis that methyl donor supplementation can prevent hepatic triglyceride accumulation induced by obesogenic diets in both sexes. Changes in liver gene expression profile and epigenetic-mediated mechanisms related to FASN DNA hypermethylation could be involved in methyl donorinduced NAFLD improvement.

Keywords Obesity - NAFLD - DNA methylation · High-fat-sucrose diet

\section{Introduction}

Non-alcoholic fatty liver disease (NAFLD) is characterized by a triglyceride accumulation in hepatocytes that can affect their normal function (Angulo 2002). NAFLD prevalence is rising in parallel with the obesity pandemic being related to cardiovascular risk (Chen et al. 2010; Edens et al. 2009) and insulin resistance (Koot et al. 2011). This disease (NAFLD) is considered the primary hepatic manifestation of metabolic syndrome, being the cause of several metabolic disorders (Fu et al. 2011). Furthermore, the increase of liver triglyceride depots can progress to non-alcoholic steatohepatitis (NASH) and later, to cirrhosis, that represents an irreversible stage that may end in hepatic carcinoma, liver transplantation, or a hepatic-related complication death (Ekstedt et al. 2006).

Besides genetic make-up, unhealthy lifestyles including physical inactivity and obesogenic diets are involved in early onset of fat accumulation in the liver (CarmielHaggai et al. 2005; Gaemers et al. 2011; Moore 2010). At this stage, adipokines such as visfatin or proinflammatory cytokines like TNF alpha, IL-6, or IL-10 (Dinant et al. 2007; Jarrar et al. 2008) as well as enzymes such as fatty 
acid synthase (FASN), one of the key metabolic genes for de novo lipogenesis, may play a role in the pathogenesis of NAFLD. In this pathway, acetyl CoA carboxylase alpha (ACACA) catalyzes the conversion of acetyl CoA to malonyl CoA and FASN catalyzes the conversion of this molecule into long chain saturated fatty acids (Strable and Ntambi 2010). Both enzymes are directly regulated by sterol regulatory elements binding protein (SREBP), which is a regulatory transcription factor (Eberle et al. 2004; Kohjima et al. 2007). Other possible mediators in liver steatosis evolution are fatty acid-binding proteins (FABP), which have roles including fatty acid uptake, transport, and metabolism, lipases, such as monoglyceride lipase (MGLL) that hydrolyzes triglycerides to fatty acids and glycerol, or chemokine ligand 2 ( $\mathrm{CCl} 2)$, a cytokine involved in the inflammatory response and atherosclerosis (Bastarrachea et al. 2007).

The research of dietary models of NAFLD and development stages in animal models include the assessment of changes by methyl donor group administration on the diet, with depletion of choline and methionine (Rinella et al. 2008; Vetelainen et al. 2007), or of vitamin $B_{12}$ and folic acid (Brunaud et al. 2003). These impairments alter the hepatic epigenetic profile and accelerate the progression from slight liver injury to cancer (Pogribny et al. 2009; Starlard-Davenport et al. 2010). On the contrary, a promethylation cocktail including choline, betaine, folic acid, and vitamin $B_{12}$ in a transgenerational model of obesity in agouti mice produced phenotypical and epigenetic alterations, where the agouti transposon was blocked and heritable obesity minimized (Waterland et al. 2008).

Previous published studies have tested methyl donor dietary supplementation in a model of obesity and NAFLD in male adults Wistar rats and have demonstrated an improvement in liver fat accumulation (Cordero et al. 2011b). The objective of this research is to describe the effects of methyl donor supplementation following an obesogenic diet in both sexes with special focus on the possible epigenetic changes induced in the methylation pattern of FASN promoter as well as gene expression of key metabolic genes in de novo lipogenesis and related to lipid metabolism in the liver.

\section{Materials and methods}

Animals, diets, and experimental design

A total of 24 male and 28 female twelve-week-old Wistar rats (initial body weight $238 \pm 5$ and $177 \pm 2 \mathrm{~g}$, respectively) supplied by the Applied Pharmacobiology Center (CIFA, Pamplona, Spain) were housed at $21-23{ }^{\circ} \mathrm{C}$ with a 12-h light cycle ( 8 am- $8 \mathrm{pm})$ and assigned to three different dietary groups: control (C, $n=7$ males and 9 females), high-fat-sucrose (HFS, $n=9$ males and 9 females), and high-fat-sucrose supplemented group (HFSsupp, $n=8$ males and 10 females) as previously described (Cordero et al. 2011b). The control group was fed a standard chow diet (2014 Teklad Global 14 \% Protein Rodent Maintenance Diet, Harlan Iberica, Barcelona, Spain) containing $20 \%$ of the energy as protein, $67 \%$ as carbohydrate, and $13 \%$ as lipid in dry weight, whereas HFS groups were fed an obesogenic diet (D12451, Research Diets, New Brunswick, NJ, USA) containing $20 \%$ of the energy as protein, $35 \%$ as total carbohydrates (17\% sucrose), and $45 \%$ as fats in dry weight. Finally, a methyl donor supplementation cocktail that contained betaine $(5 \mathrm{~g} / \mathrm{kg}$ diet, SigmaAldrich, Missouri, USA), choline (5.37 g/kg diet, SigmaAldrich, Missouri, USA), folic acid $(5.5 \mathrm{mg} / \mathrm{kg}$ diet, Sigma-Aldrich, Missouri, USA), and vitamin $B_{12}(0.5 \mathrm{mg} /$ $\mathrm{kg}$ diet, Sigma-Aldrich, Missouri, USA) was mixed with HFS powderized pellets to obtain the HFS methyl supplemented diet. In order to maintain the same diet consistency, chow and HFS pellets were also powderized for the other two dietary groups. After 8 weeks of ad libitum dietary treatment, the animals were euthanized, blood was collected, and plasma stored at $-20{ }^{\circ} \mathrm{C}$, while samples of liver, adipose depots (subcutaneous, retroperitoneal, perigonadal, and mesenteric), and gastrocnemius muscle were carefully dissected, weighed, and stored immediately at $-80^{\circ} \mathrm{C}$. All the procedures performed agreed with the National and Institutional Guidelines of the Animal Care and Use Committee at the University of Navarra.

\section{Serum measurements}

Plasma glucose (HK-CP kit; ABX diagnostic, Montpellier, France), HDL cholesterol (HDL direct CP; ABX diagnostic, Montpellier, France), lactate (L-Lactate; Randox Laboratories Ltd., Crumlin, UK), triglycerides (Triglycerides; Randox Laboratories Ltd., Crumlin, UK), and homocysteine (Homocysteine Enzymatic Assay; Demeditec Diagnostics, Kiel, Germany) levels were measured using automated COBAS MIRA equipment (Roche, Basel, Switzerland). Plasma insulin levels were determined by ELISA (Mercodia AB, Uppsala, Sweden) in automated TRITURUS equipment (Grifols International S.A., Barcelona, Spain).

Hepatic triglyceride content

Liver samples were homogenized with a Branson Sonifier 250 (Branson Ultrasonic Corporation, Danbury, USA), and triglyceride content was measured with a commercial 
reagent kit (ABX Pentra, Montpellier, France) adapted for the COBAS MIRA equipment (Roche, Basel, Switzerland).

Determination of FASN promoter methylation status

The quantitative analysis of DNA methylation in the promoter region of FASN was performed using an Sequenom EpiTyper approach (Sequenom, CA, USA), which relies on base-specific cleavage followed by MALDI-TOF mass spectrometry (Milagro et al. 2011). Briefly, genomic DNA was extracted from liver samples by using the QIAamp DNA Mini Kit (Qiagen Gmbh, Hilden, Germany) and $500 \mathrm{ng}$ were modified by using Epitec Bisulfite Kit (Qiagen Gmbh, Hilden, Germany). Primers designed for quantitative analysis of the degree of FASN promoter methylation levels were aggaagagagAAGTAAGGTTTTGAGGTTTT GGTTT for forward $\left(5^{\prime} \rightarrow 3^{\prime}\right)$ and cagtaatacgactca ctatagggagaaggctCTCAAACACCCACCCTTTCTATAAC for reverse primers $\left(5^{\prime} \rightarrow 3^{\prime}\right)$, amplifying from $-1,177$ to -688 with respect to the transcriptional start site. PCR products were excised from agarose gels, purified, treated with shrimp alkaline phosphatase to dephosphorylate unincorporated dNTPs, and later processed by MassArray Matrix Liquid Handler. Samples were heat-inactivated and subsequently incubated with Transcleave mix for concurrent in vitro transcription and base-specific cleavage. After deionization with Sequenom resin, samples were transferred to the spectroCHIP array by nanodispensation. Analysis with the Sequenom MALDI-TOF MS Compact Unit was performed following a 4-point calibration with oligonucleotides of different mass provided in the Sequenom kit. Matched peak data were exported using EpiTyper software and analyzed (CpGs shown in Fig. 2).

\section{Real-time PCR}

Total RNA was isolated from the liver according to Trizol manufacturer's instructions (Invitrogen, CA, USA) and purified with the RNeasy kit (Qiagen, MD, USA). cDNA was synthesized using $\mathrm{RT}^{2}$ Standard Kit as described by suppliers (Qiagen, MD, USA). Next, quantitative real-time PCR was performed using ABI PRISM 7900 HT Fast Real-Time PCR system (Applied Biosystems, TX, USA) with $\mathrm{RT}^{2}$ qPCR commercial primers (Qiagen, MD, USA): SREBPF1 (XM_213329), ACACA (NM_022193), FASN (NM_017332), MGLL (NM_138502), CCL2 (NM_031530), and FABP4 (NM_053365). Fold change between groups was calculated using the $2^{-\Delta \Delta \mathrm{Ct}}$ method. The gene expression levels were normalized with $\beta$-actin (ACTB, NM_031144), glyceraldehyde-3-phosphate dehydrogenase (GAPDH, NM_017008), and cyclophilin A (PPIA, NM_017101) as internal controls and using Genorm Software.
Statistical analysis

All results are expressed as mean \pm standard error (SE). Data were evaluated separately by sex using one-way ANOVA followed by a DMS post hoc test for multiple comparisons (C, HFS, HFSsupp). The level of probability was set at $p<0.05$ as statistically significant. All analyses were performed using SPSS 15.0 package for Windows (Chicago, IL, USA).

\section{Results}

As expected, HFS induced an obese phenotype in both sexes (Tables 1,2), although no relevant differences were found between the rats fed the HFS and HFSsupp diets regarding body weight gain, adiposity, or muscle and liver weights. However, there was an increase in liver fat accumulation due to HFS diet in both sexes (96\%, $p<0.01$ in males and $29 \%, p<0.05$ in females) (Fig. 1). Interestingly, the methyl donor supplementation improved this adverse dietary effect and decreased the liver triglyceride content in both males $(30 \%, p<0.05)$ and in females $(39 \%, p<0.01)$.

In this trial, the HFS diet induced a disruption of glucose homeostasis in male (Table 1) rats but not in females (Table 2). Plasma glucose $(17 \%, p<0.05)$ and insulin levels $(112 \%, p<0.001)$ were increased in males by the obesogenic diet but not in females, whereas plasma lactate levels were also higher in males fed a HFS diet $(28 \%$, $p<0.05)$ with a reversion due to the methyl supplementation $(-33 \%, p<0.05)$. In females, a HFS diet decreased cHDL levels $(-29 \%, p<0.05)$, which was reverted by the methyl donor supplementation $(34 \%, p<0.05)$.

The analysis of the DNA methylation patterns of the FASN promoter in males (Fig. 2a) showed a significant hypomethylated status $(p<0.001)$ at CpG1 in the HFS and HFSsupp groups when compared to the control group. Interestingly, CpG10-11 was hypermethylated (164\%, $p<0.01)$ in the HFSsupp as compared to control group, while the HFS group showed an intermediate value with no statistical differences with either of the other two groups. The obesogenic diet also decreased the methylation levels of CpG17-18-19 $(-50 \%, p<0.001 \mathrm{HFS}$ and $-47 \%$, $p<0.001$ HFSsupp) in males. In females (Fig. 2), CpG1 was hypomethylated by the obesogenic diet $(p<0.001)$, as opposed to the area covered by CpG9 and CpG10-11, which showed significant changes due to the HFS diet (133\%, $p<0.05$ and $104 \%, p<0.001$ ), and the methyl donor supplementation increased this dietary effect (71\%, $p<0.05$ and $34 \%, p<0.001)$, showing an additional effect between diet and methyl donor supplementation. CpG17-18-19 methylation profile was also similar to that 
Table 1 Weight-related and plasma measurements of male Wistar rats after 8 weeks of dietary treatments

\begin{tabular}{|c|c|c|c|c|}
\hline & \multicolumn{4}{|c|}{ Male dietary groups } \\
\hline & $\mathrm{C}(n=7)$ & $\operatorname{HFS}(n=9)$ & HFSsupp $(n=8)$ & ANOVA \\
\hline \multicolumn{5}{|l|}{ Weight-related measurements } \\
\hline Weight gain $(\mathrm{g})$ & $152.6 \pm 7.3 \mathrm{a}$ & $191.8 \pm 13.0 \mathrm{~b}$ & $198.8 \pm 14.0 \mathrm{~b}$ & $*$ \\
\hline Subcutaneous WAT (\%) & $1.89 \pm 0.09 \mathrm{a}$ & $3.14 \pm 0.19 b$ & $3.10 \pm 0.13 b$ & $* * *$ \\
\hline Retroperitoneal WAT (\%) & $2.83 \pm 0.25 \mathrm{a}$ & $3.65 \pm 0.16 b$ & $3.72 \pm 0.21 b$ & $*$ \\
\hline Epididymal WAT (\%) & $2.32 \pm 0.20 \mathrm{a}$ & $3.10 \pm 0.11 b$ & $3.32 \pm 0.20 b$ & $* *$ \\
\hline Mesenteric WAT (\%) & $1.06 \pm 0.11 \mathrm{a}$ & $1.45 \pm 0.09 b$ & $1.45 \pm 0.13 b$ & $*$ \\
\hline Liver $(\%)$ & $2.32 \pm 0.03 \mathrm{a}$ & $2.37 \pm 0.06 \mathrm{a}$ & $2.25 \pm 0.05 \mathrm{a}$ & $\mathrm{ns}$ \\
\hline Gastrocnemius muscle $(\%)$ & $0.54 \pm 0.02 \mathrm{a}$ & $0.51 \pm 0.01 \mathrm{a}$ & $0.51 \pm 0.01 \mathrm{a}$ & $\mathrm{ns}$ \\
\hline \multicolumn{5}{|l|}{ Plasma values } \\
\hline Glucose (mg/dL) & $114.5 \pm 5.3 \mathrm{a}$ & $134.0 \pm 4.7 b$ & $122.9 \pm 5.4 \mathrm{a}, \mathrm{b}$ & $*$ \\
\hline HDL cholesterol (mg/dL) & $69.2 \pm 3.8 \mathrm{a}$ & $72.0 \pm 2.8 \mathrm{a}$ & $79.6 \pm 3.0 \mathrm{a}$ & ns \\
\hline Triglycerides (mg/dL) & $108.4 \pm 8.8 \mathrm{a}$ & $100.0 \pm 11.3 \mathrm{a}$ & $103.1 \pm 9.6 \mathrm{a}$ & $\mathrm{ns}$ \\
\hline Lactate $(\mathrm{mg} / \mathrm{dL})$ & $25.6 \pm 3.2 \mathrm{a}, \mathrm{b}$ & $32.8 \pm 3.2 \mathrm{a}$ & $22.1 \pm 1.1 b$ & $*$ \\
\hline Homocysteine $(\mu \mathrm{mol} / \mathrm{L})$ & $17.9 \pm 1.0 \mathrm{a}$ & $18.7 \pm 3.1 \mathrm{a}$ & $14.3 \pm 1.0 \mathrm{a}$ & ns \\
\hline Insulin $(\mu \mathrm{U} / \mathrm{mL})$ & $20.3 \pm 2.4 \mathrm{a}$ & $43.1 \pm 5.0 \mathrm{~b}$ & $46.7 \pm 3.6 b$ & $* * *$ \\
\hline
\end{tabular}

All the results are expressed as the mean \pm SE. Statistical analyses were performed using one-way Anova and Tukey post hoc test. Different letters $(\mathrm{a}, \mathrm{b})$ indicate significant differences between groups of at least $p<0.05$

WAT white adipose tissue, $C$ control group, HFS high-fat-sucrose group, HFSsupp high-fat-sucrose supplemented with methyl donors group, $n s$ non statistically significant

$* p<0.05, * * p<0.01, * * * p<0.001$

Table 2 Weight-related and plasma measurements of female Wistar rats after 8 weeks of dietary treatments

\begin{tabular}{|c|c|c|c|c|}
\hline & \multicolumn{4}{|c|}{ Female dietary groups } \\
\hline & $\mathrm{C}(n=7)$ & $\operatorname{HFS}(n=9)$ & HFSsupp $(n=10)$ & ANOVA \\
\hline \multicolumn{5}{|l|}{ Weight-related measurements } \\
\hline Weight gain (g) & $68.7 \pm 5.9 \mathrm{a}$ & $102.8 \pm 8.9 b$ & $109.4 \pm 11.4 \mathrm{~b}$ & $*$ \\
\hline Subcutaneous WAT ( \%) & $1.40 \pm 0.14 \mathrm{a}$ & $2.02 \pm 0.26 \mathrm{a}, \mathrm{b}$ & $2.27 \pm 0.28 b$ & $*$ \\
\hline Retroperitoneal WAT ( \%) & $1.87 \pm 0.19 \mathrm{a}$ & $2.70 \pm 0.29 b$ & $3.09 \pm 0.24 b$ & $* *$ \\
\hline Epididymal WAT (\%) & $2.42 \pm 0.30 \mathrm{a}$ & $3.53 \pm 0.49 \mathrm{a}$ & $3.84 \pm 0.41 \mathrm{a}$ & ns \\
\hline Mesenteric WAT (\%) & $1.28 \pm 0.18 \mathrm{a}$ & $1.63 \pm 0.19 a$ & $1.62 \pm 0.21 \mathrm{a}$ & ns \\
\hline Liver $(\%)$ & $2.18 \pm 0.03 \mathrm{a}$ & $2.14 \pm 0.06 a$ & $2.24 \pm 0.07 \mathrm{a}$ & ns \\
\hline Gastrocnemius muscle $(\%)$ & $0.59 \pm 0.02 \mathrm{a}$ & $0.55 \pm 0.03 \mathrm{a}$ & $0.51 \pm 0.04 \mathrm{a}$ & $\mathrm{ns}$ \\
\hline \multicolumn{5}{|l|}{ Plasma values } \\
\hline Glucose (mg/dL) & $103.4 \pm 6.1 \mathrm{a}$ & $108.9 \pm 3.7 \mathrm{a}$ & $119.3 \pm 6.7 \mathrm{a}$ & ns \\
\hline HDL cholesterol (mg/dL) & $56.8 \pm 4.3 \mathrm{a}$ & $40.4 \pm 4.6 b$ & $54.2 \pm 4.5 \mathrm{a}$ & $*$ \\
\hline Triglycerides (mg/dL) & $86.5 \pm 6.9 a$ & $57.7 \pm 5.3 \mathrm{a}$ & $74.1 \pm 13.2 \mathrm{a}$ & ns \\
\hline Lactate $(\mathrm{mg} / \mathrm{dL})$ & $27.8 \pm 4.3 \mathrm{a}$ & $26.1 \pm 3.4 \mathrm{a}$ & $34.3 \pm 4.7 \mathrm{a}$ & $\mathrm{ns}$ \\
\hline Homocysteine $(\mu \mathrm{mol} / \mathrm{L})$ & $15.6 \pm 1.1 \mathrm{a}$ & $15.2 \pm 0.8 \mathrm{a}$ & $14.1 \pm 0.9 \mathrm{a}$ & ns \\
\hline Insulin $(\mu \mathrm{U} / \mathrm{mL})$ & $15.5 \pm 4.7 \mathrm{a}$ & $16.4 \pm 3.3 \mathrm{a}$ & $21.2 \pm 4.5 \mathrm{a}$ & ns \\
\hline
\end{tabular}

All the results are expressed as the mean \pm SE. Statistical analyses were performed using one-way Anova and Tukey post hoc test. Different letters $(\mathrm{a}, \mathrm{b})$ indicate significant differences between groups of at least $p<0.05$

WAT white adipose tissue, $C$ control group, HFS high-fat-sucrose group, HFSsupp high-fat-sucrose supplemented with methyl donors group, $n s$ non statistically significant

$* p<0.05, * * p<0.01$ 


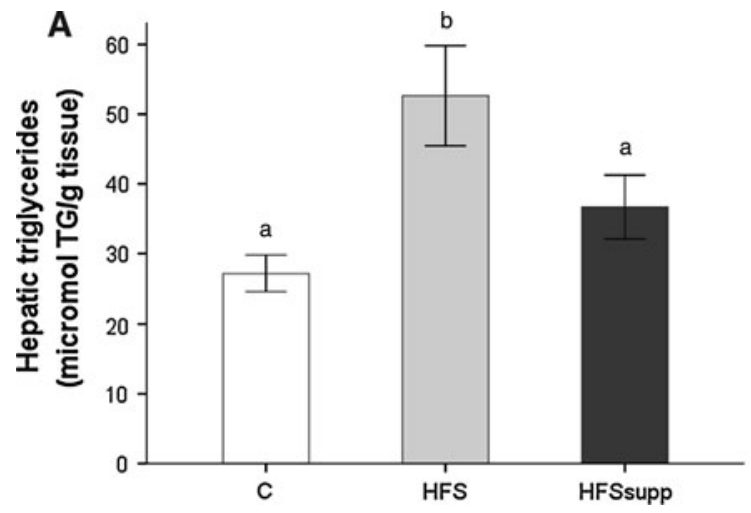

Fig. 1 Hepatic triglyceride content (in $\mu$ mol of triglycerides per gram of tissue) in (a) male and (b) female Wistar rats. Different letters indicate significant differences between groups of at least $p<0.05$

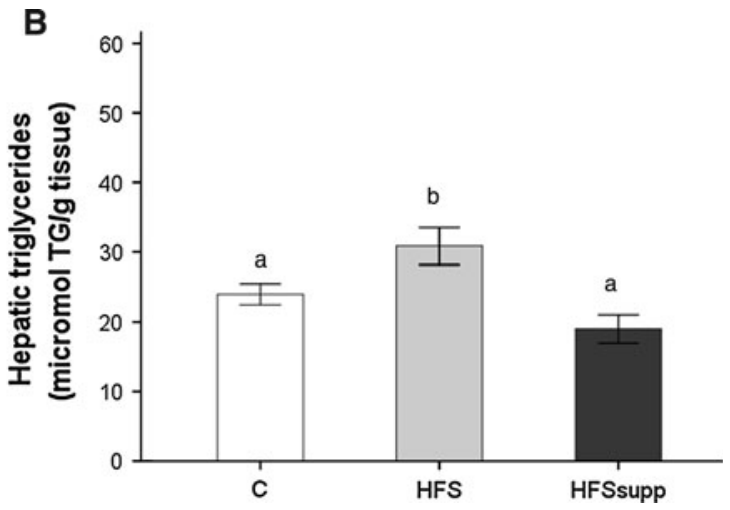

(c control group-white, HFS high-fat-sucrose group-grey, HFSsupp high-fat-sucrose supplemented with methyl donors groupblack)
Fig. 2 Quantitative DNA methylation analysis using MassARRAY system for FASN promoter in liver tissue from (a) male and (b) female Wistar rats (TSS transcriptional start site). The results are shown as percentages of methylation. All results are expressed as mean \pm SD. Different letters indicate significant differences between groups of at least $p<0.05$ (Control group, white; high-fat-sucrose group, grey; high-fat-sucrose supplemented with methyl donors group; black; **p $<0.01$, $* * * p<0.001)$

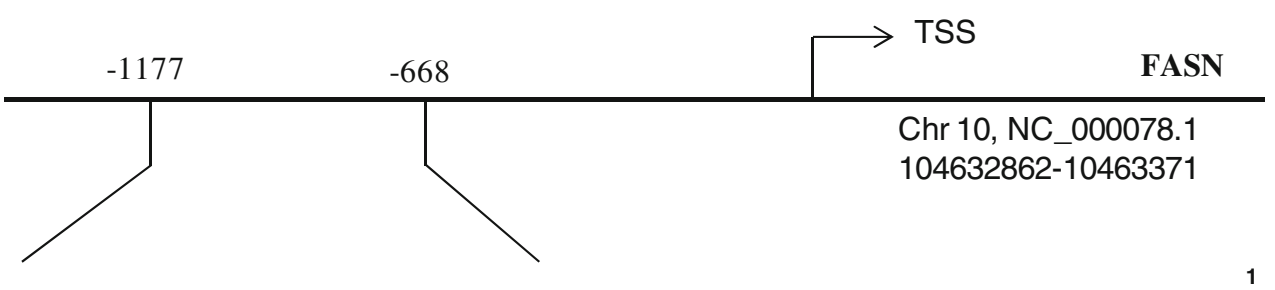

GGGTTGACAAGCAAGGCTCTGAGGCTCTGGCTTTTGCTATAGACATGCAGTCAAGGACCACTGACACTACTCCCTCCTTAGCG CCCCCCCCCCCAAAGCCACTGCCCATAAGGTTGGTCTTAGTGGCCTGGGCCTGTAGTGGAAGGGCAGAAGGAAAGGGATCA 2 ACTCTGAGCAGTCTGTGTCTITTGGTCGGTGAGTTTTCATCATCTCCCGTCCCCAAATTCGATAACCCTTTCAAAAGAGGAAT TTAAAGGAGGGAGGGTGAGGGTCCCGGAACCAGCAACTCAGGGAGG $\mathbf{6} \mathbf{7} \mathbf{7} \mathbf{8}$ $\begin{array}{llllll}10 & 11 & 1213 & 1516 & 1718 & 19\end{array}$ GGGAGGGGTGGTATCCCGCTCGCCAGATGGCCGCGCCTGGACACTGAACGGACTCAGGAGACCGCGGCACGCGCCCGTCA 20 21

GTGTTCCCTATCCTGCCTACTGCTCTCGTCCCTGCCCGCATCCTGGTCTCCAAGGCGGCCACAGAAAGGGTGGGTGTCTGAG
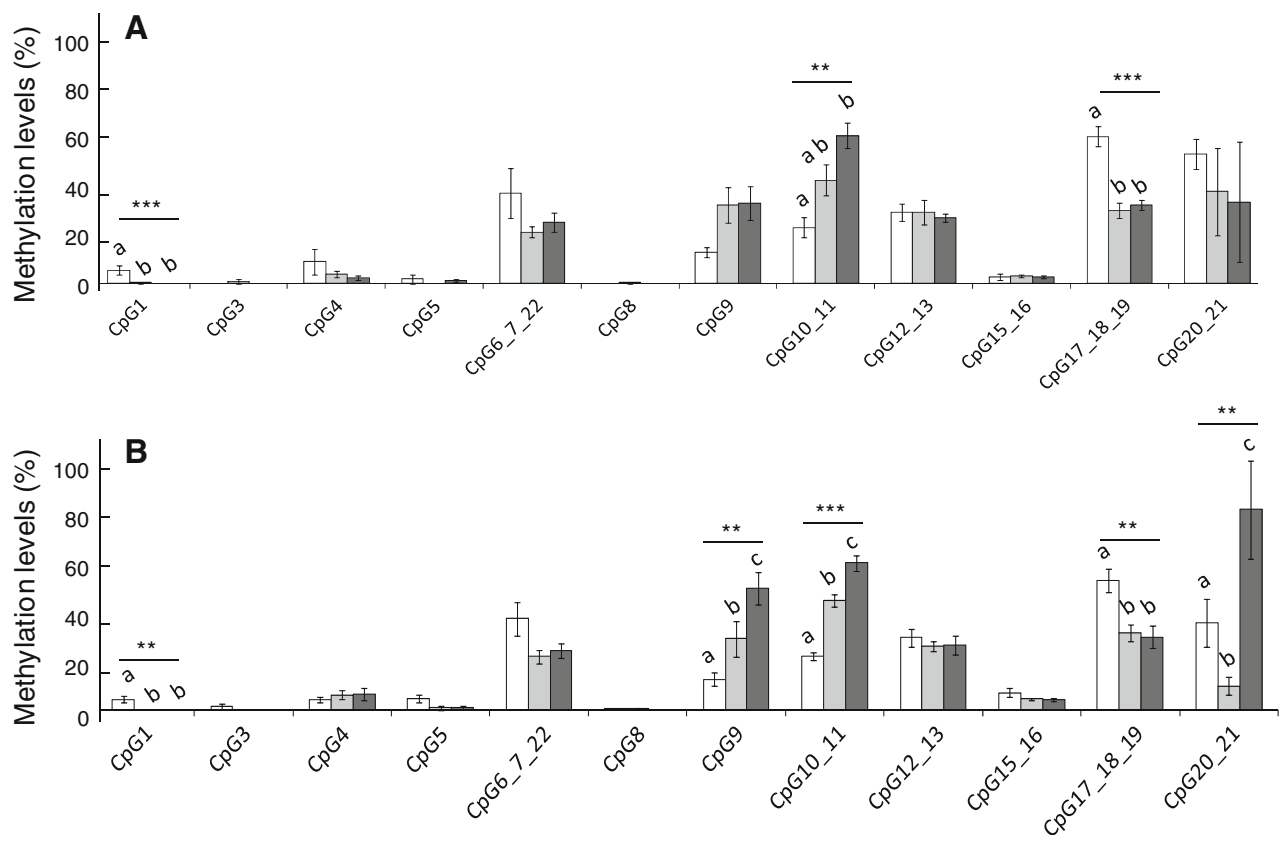
presented in males, with a hypomethylation due to the obesogenic diet $(-40 \%, p<0.01$ HFS and $-44 \%$, $p<0.01$ HFSsupp). There was also a hypermethylation of CpG20-21 induced by the methyl donor supplementation when compared to both $\mathrm{C}$ and HFS diets (767\%, $p<0.001)$, which showed a lower methylation percentage than the control group $(-72 \%, p<0.01)$. Finally, in females, promoter methylation mean values did not change due to diet but presented a significative increase (40\%, $p<0.05)$ when comparing HFS with HFS supplemented diet.

Additionally, the hepatic expression of some key genes involved in lipid metabolism-related genes was analyzed by RT-PCR in male Wistar rats (Fig. 3). Regarding de novo lipogenesis pathway, significant changes were found in ACACA expression $(p<0.01)$, which was $66 \%$ higher due to methyl donor supplementation. Whereas, SREBPF1 and FASN only showed a trend to increase $(p=0.095$ and $p=0.082)$. The cytokine CCL2 was markedly downregulated $(-76 \%, p<0.05)$ by methyl supplementation when compared to controls, with no significant changes induced by HFS diet. The lipolytic enzyme MGLL showed a decrease in the mRNA expression levels in rats fed the obesogenic diet $(-30 \%, p<0.05)$. Finally, FABP4 did not show significant differences $(p=0.093)$ between the experimental dietary groups.

\section{Discussion}

NAFLD is the main early liver manifestation of the metabolic syndrome and has a close association with obesity (Moore 2010). The increased fat depots tend to expand to the visceral organs affecting their functionality (Guri and
Bassaganya-Riera 2011). In this way, this research has been designed to be focused on the initial stage of liver damage, NAFLD, since it could potentially be easier to treat or revert than more advanced stages such as NASH or cirrhosis. For that reason, it was decided to supplement a HFS diet for a period of eight weeks, when obese phenotype was at the beginning (Cordero et al. 2011b; Milagro et al. 2006). We analyzed all variables according to the sex, as gender is a factor that appears to confer differential vulnerability to obesogenic diets (Clark et al. 2011), since many of the relevant studies in this field have been conducted only in males. In this way, previous investigations have described that obesity-linked disorders such as insulin and glucose homeostasis or fat depot distributions differ between sexes (Priego et al. 2008). It is also known that steroid hormones can play a role in metabolism, accumulation, and distribution of the different adipose depots (Mayes and Watson 2004). Thus, we decided to analyze in a separate way males and females, and also to avoid possible disturbances and errors derived from hormonal status, we determined gene expression only in male rats.

Biochemical variables were affected by an obesogenic diet and methyl donor supplementation. In males, the increase in insulin levels due to the HFS diet (Boque et al. 2009) was not enough to control glucose circulating levels. Methyl donor supplemented groups presented an intermediate value that suggests a partial protection of these methyl substrates on glucose homeostasis. In this sense, previous studies during the perinatal nutrition period have shown that maternal folic acid supplementation decreases glucose levels in the offspring (Chmurzynska et al. 2011), while its depletion, together with low choline levels, leads to increased plasma glucose levels (Maloney et al. 2009). However, other studies have reported that hepatic steatosis

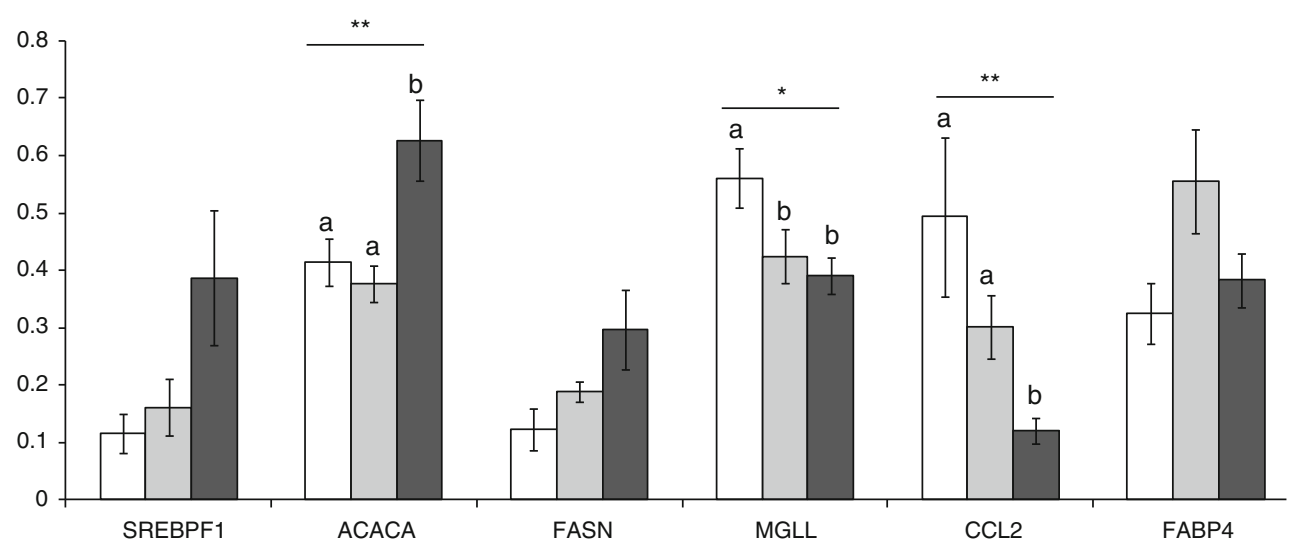

Fig. 3 Expression levels (mRNA) of SREBPF1, CACA, FASN, MGLL, CCL2 and FABP4 measured by RT-PCR in liver from C, HFS and HFSsupp groups in male Wistar rats. Data are presented as mean \pm SE measured in arbitrary units after normalization with ACTB, GAPDH and PPIA. Different letters in the same gene indicate statistically significant differences between groups of at least $p<0.05$. (control group, white; high-fat-sucrose group, grey; highfat-sucrose supplemented with methyl donors group, black; $* p<0.05, * * p<0.01)$ 
could be associated to increased lactate levels (Kalhan et al. 2011). Lactate can be used as a substrate activating liver gluconeogenesis, which may contribute to increase glucose levels. However, HDL cholesterol, an atherogenic protective factor, is decreased in animals fed obesogenic and sucrose enriched diets (Kamgang et al. 2005) being known that this molecule removes cholesterol from the peripheral tissues transporting it to the liver to be metabolized (Tall 2008). Interestingly, in this study, a methyl donor cocktail can produce a beneficial cardiovascular effect in female Wistar rats by increasing cHDL plasma levels during an obesogenic-proatherogenic dietary treatment.

The dietary depletion of some micronutrients such as choline or betaine has been repeatedly used to generate models of hepatic cirrhosis (Pogribny et al. 2009; Tryndyak et al. 2011; Vetelainen et al. 2007), and betaine supplementation has been studied as a potential hepatoprotective substrate (Kathirvel et al. 2010). Folic acid and vitamin $B_{12}$ have mainly been studied in periconceptual periods to prevent neural tube defects and future congenital diseases (Ba et al. 2011; Chmurzynska et al. 2011; Dhobale and Joshi 2011; Wilson et al. 2007). The effects of these two promethylating vitamins are related to epigenetic changes in the offspring due to the accessibility of the DNA during early development (Ozanne and Constancia 2007). The supplementation cocktail we used was tested in agouti mice, and it was found that the agouti transposon was blocked by epigenetic silencing, whereas transgenerational heritability of obesity and skin color was interrupted (Waterland et al. 2008). A previous investigation of our group (Cordero et al. 2011b) reported that the diet supplementation of promethyling agents during adulthood had a positive impact effect on hepatic triglyceride accumulation. According to the biochemical onset of this disease, different studies have reported that changes in epigenetic marks could be related to liver damage. For example, insulin resistance levels in patient with NAFLD showed a relation with PPARgamma hepatic methylation levels (Sookoian et al. 2010), and a maternal low protein intake is related to changes in Igf2 methylation levels in the offspring (Gong et al. 2010). On the other hand, obesogenic diets also have the potential to modify the epigenome (Campion et al. 2009), mainly during perinatal periods (Martinez et al. 2012; Vucetic et al. 2010), but also in adulthood (Lomba et al. 2010). On the contrary, the epigenetic pattern may also act as a response element for dietary treatments (Cordero et al. 2011a; Milagro et al. 2011). In this context, changes in FASN methylation in epididymal adipose tissue induced by the intake of an obesogenic diet have been reported in Wistar rats (Lomba et al. 2010). Our results concerning this key enzyme of de novo lipogenesis evidenced changes in its methylation pattern induced by an obesogenic diet, but also by the methyl donor supplementation.

On the other hand, in NAFLD stage, the liver increases the uptake and synthesis of fat (Angulo 2002). Thus, obese rats overexpress genes involved in de novo lipogenesis, including SREBPF1, ACACA, and FASN. SREBPF1 is an activator of many enzymes from this pathway and also for ACACA and FASN (Kohjima et al. 2007). In this research, we found a correlation (Electronic Supplementary Material, Fig. 4) between SREBPF1 expression and ACACA $(p<0.05, r=0.477)$ and also with FASN $(p<0.05$, $r=0.463)$. These outcomes concluded that lipid synthesis in the liver is increased by the obesogenic diet and also by methyl donor supplementation. On the other hand, MGLL lipase is involved in the activation of intracellular triglyceride hydrolysis to fatty acids and glycerol. In our study, MGLL expression profile showed a decrease attributable to HFS effect. The impact on lipogenic and lipolytic pathways suggests that methyl donor supplementation of a HFS diet increases the total amount of triglycerides produced by the liver decreasing their accumulation, so the explanation could be described in a lipid storage-mobilization and lipolysis more than on synthesis. However, liver FFA uptake is also increased in metabolic syndrome and obesity, in such a way that this situation affects their accumulation in the liver. The analysis of FABP4 expression levels, a protein implicated in fatty acid uptake and upregulated in metabolic syndrome and obesity (Terra et al. 2011), revealed no statistical differences. This FABP isoform is highly expressed in adipose tissue, but it has been also associated in liver with fat accumulation in this organ (Westerbacka et al. 2007). Curiously in our study, the obesogenic diet induced a trend to increase FABP4 gene expression $(p=0.093)$ that was reverted by methyl donor supplementation. Finally, although we described differences in NAFLD gene expression profile, the pathways that unravel the decrease showed in hepatic triglycerides content are still uncertain being necessary other studies to deepen into these mechanisms.

Contrary as expected, methylation levels in FASN promoter did not correlate with its gene expression levels. It should be kept in mind that the expression of this gene is not only regulated by DNA methylation; there are other epigenetic mechanisms such as histone modifications or microRNA regulation that could affect mRNA levels (Campion et al. 2009).

It appears that methyl donors do not influence fat accumulation in adipose tissue, but it seems that methyl donors affect only the fat accumulation in liver (Perseghin 2011). The treatment with methyl donors may not be effective against obesity itself, but this dietary supplementation could decrease the prevalence of NAFLD as the main hepatic manifestation of metabolic syndrome, with all 
the comorbidities associated to this disease including insulin resistance. In this way, even if this work could be an useful tool in the treatment of NAFLD, there are some limitations that researchers have to evaluate in a cautious and appropriate manner, such as the number of individuals, the measurement of gene expression only in males or the possibility to include other technique in order to validate Sequenom results.

Summing up, supplementation with methyl donor groups in obesogenic diets reverted NAFLD development and associated features in both sexes. One possible mechanism involved in this alteration is by affecting the epigenetic machinery and by inducing DNA hypermethylation or hypomethylation. Indeed, changes in DNA methylation patterns on the FASN gene promoter were influenced by the obesogenic dietary intake and also with the dietary supplementation with methyl donors.

Acknowledgments The technical assistance of V. Fernandez is gratefully acknowledged as well as Alexandra Claire Simpson for checking the manuscript spelling and grammar for English. "Linea Especial (LE/97)" from University of Navarra for financial support. P. Cordero and A. M. Gomez-Uriz were recipients of predoctoral fellowships from "Asociacion de Amigos de la Universidad de Navarra". Also RETICS and CIBERobn schemes from Carlos III Institute are gratefully acknowledged.

Conflict of interest The authors declare no conflict of interest.

Ethical standards All animal manipulations were made in accordance with European Community Guidelines and the University of Navarra Ethical Committee for the use of laboratory animals.

\section{References}

Angulo P (2002) Nonalcoholic fatty liver disease. N Engl J Med 346:1221-1231

Ba Y, Yu H, Liu F et al (2011) Relationship of folate, vitamin B12 and methylation of insulin-like growth factor-II in maternal and cord blood. Eur J Clin Nutr 65:480-485. doi:410.1038/ejen. 2010.1294

Bastarrachea RA, López-Alvarenga JC, Bolado-García VE, TéllezMendoza J, Laviada-Molina H, Comuzzie AG (2007) Macrophages, inflammation, adipose tissue, obesity and insulin resistance. Gac Med Mex 143:505-512

Boque N, Campion J, Paternain L et al (2009) Influence of dietary macronutrient composition on adiposity and cellularity of different fat depots in Wistar rats. J Physiol Biochem 65:387395. doi:10.1007/BF03185934

Brunaud L, Alberto JM, Ayav A et al (2003) Effects of vitamin B12 and folate deficiencies on DNA methylation and carcinogenesis in rat liver. Clin Chem Lab Med 41:1012-1019. doi:1010.1515/ CCLM.2003.1155

Campion J, Milagro FI, Martinez JA (2009) Individuality and epigenetics in obesity. Obes Rev 10:383-392. doi:310.1111/ j.1467-1789X.2009.00595.x

Carmiel-Haggai M, Cederbaum AI, Nieto N (2005) A high-fat diet leads to the progression of non-alcoholic fatty liver disease in obese rats. Faseb J 19:136-138. doi:110.1096/fj.1004-2291fje
Chen CH, Nien CK, Yang CC, Yeh YH (2010) Association between nonalcoholic fatty liver disease and coronary artery calcification. Dig Dis Sci 55:1752-1760. doi:1710.1007/s10620-1000910935-10629

Chmurzynska A, Stachowiak M, Gawecki J, Pruszynska-Oszmalek E, Tubacka M (2011) Protein and folic acid content in the maternal diet determine lipid metabolism and response to high-fat feeding in rat progeny in an age-dependent manner. Genes Nutr. doi: 10.1007/s12263-12011-10253-12267

Clark C, Smith W, Lochner A, du Toit EF (2011) The effects of gender and obesity on myocardial tolerance to ischemia. Physiol Res 60:291-301

Cordero P, Campion J, Milagro FI et al (2011a) Leptin and TNFalpha promoter methylation levels measured by MSP could predict the response to a low-calorie diet. J Physiol Biochem 67:463-470. doi:410.1007/s13105-13011-10084-13104

Cordero P, Campion J, Milagro FI, Martinez JA (2011b) Dietary supplementation with methyl donor groups could prevent nonalcoholic fatty liver. Hepatology 53:2151-2152. doi:2110. 1002/hep.24164

Dhobale M, Joshi S (2011) Altered maternal micronutrients (folic acid, vitamin $\mathrm{B}(12)$ ) and omega 3 fatty acids through oxidative stress may reduce neurotrophic factors in preterm pregnancy. J Matern Fetal Neonatal Med. doi:10.3109/14767058.1476 2011.14579209

Dinant S, Vetelainen RL, Florquin S, van Vliet AK, van Gulik TM (2007) IL-10 attenuates hepatic I/R injury and promotes hepatocyte proliferation. J Surg Res 141:176-182. doi:110.1016/ j.jss.2006.1009.1018

Eberle D, Hegarty B, Bossard P, Ferre P, Foufelle F (2004) SREBP transcription factors: master regulators of lipid homeostasis. Biochimie 86:839-848. doi:810.1016/j.biochi.2004.1009.1018

Edens MA, Kuipers F, Stolk RP (2009) Non-alcoholic fatty liver disease is associated with cardiovascular disease risk markers. Obes Rev 10:412-419. doi:410.1111/j.1467-1789X.2009.00594.x

Ekstedt M, Franzen LE, Mathiesen UL et al (2006) Long-term followup of patients with NAFLD and elevated liver enzymes. Hepatology 44:865-873. doi:810.1002/hep.21327

Fu JF, Shi HB, Liu LR et al (2011) Non-alcoholic fatty liver disease: an early mediator predicting metabolic syndrome in obese children? World J Gastroenterol 17:735-742. doi:710.3748/wjg. v3717.i3746.3735

Gaemers IC, Stallen JM, Kunne C et al (2011) Lipotoxicity and steatohepatitis in an overfed mouse model for non-alcoholic fatty liver disease. Biochim Biophys Acta 1812:447-458. doi:410.1016/ j.bbadis.2011.1001.1003

Gong L, Pan YX, Chen H (2010) Gestational low protein diet in the rat mediates Igf2 gene expression in male offspring via altered hepatic DNA methylation. Epigenetics 5:619-626. doi:610.4161/ epi.4165.4167.12882

Guri AJ, Bassaganya-Riera J (2011) Systemic effects of white adipose tissue dysregulation and obesity-related inflammation. Obesity (Silver Spring) 19:689-700. doi:610.1038/oby.2010.1230

Jarrar MH, Baranova A, Collantes R et al (2008) Adipokines and cytokines in non-alcoholic fatty liver disease. Aliment Pharmacol Ther 27:412-421. doi:410.1111/j.1365-2036.2007.03586.x

Kalhan SC, Guo L, Edmison J et al (2011) Plasma metabolomic profile in nonalcoholic fatty liver disease. Metabolism 60:404413. doi:410.1016/j.metabol.2010.1003.1006

Kamgang R, Mboumi RY, N'Dille GP, Yonkeu JN (2005) Cameroon local diet-induced glucose intolerance and dyslipidemia in adult Wistar rat. Diabetes Res Clin Pract 69:224-230. doi:210.1016/ j.diabres.2005.1002.1005

Kathirvel E, Morgan K, Nandgiri G et al (2010) Betaine improves nonalcoholic fatty liver and associated hepatic insulin resistance: a potential mechanism for hepatoprotection by betaine. Am J 
Physiol Gastrointest Liver Physiol 299:G1068-G1077. doi: 1010.1016/j.diabres.2005.1002.1005

Kohjima M, Enjoji M, Higuchi N et al (2007) Re-evaluation of fatty acid metabolism-related gene expression in nonalcoholic fatty liver disease. Int J Mol Med 20:351-358

Koot BG, van der Baan-Slootweg OH, Tamminga-Smeulders CL et al (2011) Lifestyle intervention for non-alcoholic fatty liver disease: prospective cohort study of its efficacy and factors related to improvement. Arch Dis Child 96:669-674. doi: 10.1136/adc.2010.199760

Lomba A, Martinez JA, Garcia-Diaz DF et al (2010) Weight gain induced by an isocaloric pair-fed high fat diet: a nutriepigenetic study on FASN and NDUFB6 gene promoters. Mol Genet Metab 101:273-278. doi:210.1016/j.ymgme.2010.1007.1017

Maloney CA, Hay SM, Rees WD (2009) The effects of feeding rats diets deficient in folic acid and related methyl donors on the blood pressure and glucose tolerance of the offspring. Br J Nutr 101:1333-1340. doi:1310.1017/S0007114508066798

Martinez JA, Cordero P, Campion J, Milagro FI (2012) Interplay of early-life nutritional programming on obesity, inflammation and epigenetic outcomes. Proc Nutr Soc 71:276-283. doi:10.1017/ S0029665112000055

Mayes JS, Watson GH (2004) Direct effects of sex steroid hormones on adipose tissues and obesity. Obes Rev 5:197-216. doi:110. 1111/j.1467-1789X.2004.00152.x

Milagro FI, Campion J, Martinez JA (2006) Weight gain induced by high-fat feeding involves increased liver oxidative stress. Obesity (Silver Spring) 14:1118-1123. doi:1110.1038/oby. 2006.1128

Milagro FI, Campion J, Cordero P et al (2011) A dual epigenomic approach for the search of obesity biomarkers: DNA methylation in relation to diet-induced weight loss. Faseb J 25:1378-1389. doi:1310.1096/fj.1310-170365

Moore JB (2010) Non-alcoholic fatty liver disease: the hepatic consequence of obesity and the metabolic syndrome. Proc Nutr Soc 69:211-220. doi:210.1017/S0029665110000030

Ozanne SE, Constancia M (2007) Mechanisms of disease: the developmental origins of disease and the role of the epigenotype. Nat Clin Pract Endocrinol Metab 3:539-546. doi:510.1038/ ncpendmet0531

Perseghin G (2011) Lipids in the wrong place: visceral fat and nonalcoholic steatohepatitis. Diabetes Care 34(Suppl 2):S367S370. doi:310.2337/dc2311-s2249

Pogribny IP, Tryndyak VP, Bagnyukova TV et al (2009) Hepatic epigenetic phenotype predetermines individual susceptibility to hepatic steatosis in mice fed a lipogenic methyl-deficient diet. J Hepatol 51:176-186. doi:110.1016/j.jhep.2009.1003.1021

Priego T, Sanchez J, Pico C, Palou A (2008) Sex-differential expression of metabolism-related genes in response to a high-fat diet. Obesity (Silver Spring) 16:819-826. doi:810.1038/oby. 2007.1117
Rinella ME, Elias MS, Smolak RR, Fu T, Borensztajn J, Green RM (2008) Mechanisms of hepatic steatosis in mice fed a lipogenic methionine choline-deficient diet. J Lipid Res 49:1068-1076. doi:1010.1194/jlr.M800042-JLR800200

Sookoian S, Gianotti TF, Rosselli MS, Burgueno AL, Castano GO, Pirola CJ (2010) Liver transcriptional profile of atherosclerosisrelated genes in human nonalcoholic fatty liver disease. Atherosclerosis 218:378-385. doi:310.1016/j.atherosclerosis.2011. 1005.1014

Starlard-Davenport A, Tryndyak V, Kosyk O et al (2010) Dietary methyl deficiency, microRNA expression and susceptibility to liver carcinogenesis. J Nutrigenet Nutrigenomics 3:259-266. doi:210.1159/000324362

Strable MS, Ntambi JM (2010) Genetic control of de novo lipogenesis: role in diet-induced obesity. Crit Rev Biochem Mol Biol 45:199-214. doi:110.3109/10409231003667500

Tall AR (2008) Cholesterol efflux pathways and other potential mechanisms involved in the athero-protective effect of high density lipoproteins. J Intern Med 263:256-273. doi:210.1111/ j.1365-2796.2007.01898.x

Terra X, Quintero Y, Auguet T et al (2011) FABP 4 is associated with inflammatory markers and metabolic syndrome in morbidly obese women. Eur J Endocrinol 164:539-547. doi:510.1530/ EJE-1510-1195

Tryndyak VP, Han T, Muskhelishvili L et al (2011) Coupling global methylation and gene expression profiles reveal key pathophysiological events in liver injury induced by a methyl-deficient diet. Mol Nutr Food Res 55:411-418. doi:410.1002/mnfr.2010 00300

Vetelainen R, van Vliet A, van Gulik TM (2007) Essential pathogenic and metabolic differences in steatosis induced by choline or methione-choline deficient diets in a rat model. J Gastroenterol Hepatol 22:1526-1533. doi:1510.1111/j.1440-1746.2006.04701.x

Vucetic Z, Kimmel J, Totoki K, Hollenbeck E, Reyes TM (2010) Maternal high-fat diet alters methylation and gene expression of dopamine and opioid-related genes. Endocrinology 151:47564764. doi:4710.1210/en.2010-0505

Waterland RA, Travisano M, Tahiliani KG, Rached MT, Mirza S (2008) Methyl donor supplementation prevents transgenerational amplification of obesity. Int J Obes (Lond) 32:1373-1379. doi: 1310.1038/ijo.2008.1100

Westerbacka J, Kolak M, Kiviluoto T et al (2007) Genes involved in fatty acid partitioning and binding, lipolysis, monocyte/macrophage recruitment, and inflammation are overexpressed in the human fatty liver of insulin-resistant subjects. Diabetes 56:2759-2765. doi:10.2337/db07-0156

Wilson RD, Johnson JA, Wyatt P et al (2007) Pre-conceptional vitamin/folic acid supplementation 2007: the use of folic acid in combination with a multivitamin supplement for the prevention of neural tube defects and other congenital anomalies. J Obstet Gynaecol Can 29:1003-1026 